\title{
On electric vehicle battery charger modeling
}

\begin{abstract}
The increase of electric vehicle (EV) battery chargers connected to electric networks could lead to future harmonic problems in power systems. These loads are nonlinear devices that inject harmonic currents and pollute network voltages. Thus, battery charger modeling must be studied in detail to determine their harmonic emissions and prevent future problems. This paper investigates EV battery charger behavior, analyzes its equivalent circuit and reports a model for each conduction mode of the circuit. Experimental measurements and PSpice numerical simulations support the study.
\end{abstract}

L. Sainz ${ }^{1}$, J. J. Mesas ${ }^{1}$ and J. Balcells ${ }^{2}$

${ }^{1}$ Department of Electrical Engineering

E.T.S.E.I.B, Technical University of Catalonia (UPC)

Av. Diagonal 647, 08028 Barcelona (Spain)

Phone/Fax number:+003493 4011759/+003493 4017433e-mails: sainz@ee.upc.edu, juan.jose.mesas@upc.edu

2 Department of Electronics Engineering

E.T.S.E.I.T, Technical University of Catalonia (UPC)

Colom 1, 08222 Terrassa (Spain)

Phone/Fax number:+0034 93 7398193/+0034 93 7398016, e-mail: josep.balcells@upc.edu

\section{Key words}

Battery chargers, harmonics, power quality.

\section{Introduction}

Effective transportation systems are essential for developed countries' prosperity, having significant impacts on economic growth, social development and the environment. In 2004 the transport sector consumed 30\% of the total final energy consumption and it was responsible for $25 \%$ of $\mathrm{CO} 2$ emissions (EU-25) [1].

Electrical vehicles (EV) have recently emerged as a promising alternative that uses electricity to displace a significant fraction of fleet petroleum consumption. Nevertheless, this new situation and the expected future growth of EVs can create serious problems of power quality in existing grids, mainly where parking installations are concentrated [2]-[3]. Thus the growing presence of EV battery chargers in residential installations could increase the harmonic levels in power distribution systems. For this reason, several studies in the literature attempt to predict the harmonic impact of these non-linear loads on the network and investigate the harmonic emissions of battery chargers and clusters of these loads in the network [4]-[8]. Others focus on the effects of these devices on the system [9]-[12].

The paper presents the equivalent circuit of EV battery chargers and studies its conduction modes. The analytical models of the battery charger conduction modes are developed in detail. Experimental and numerical examples of these modes are also reported.

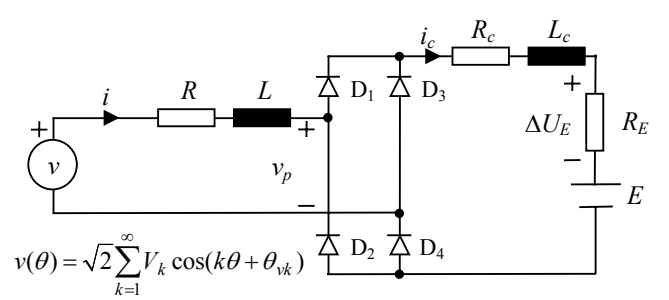

Fig. 1. Equivalent circuit of EV battery chargers.
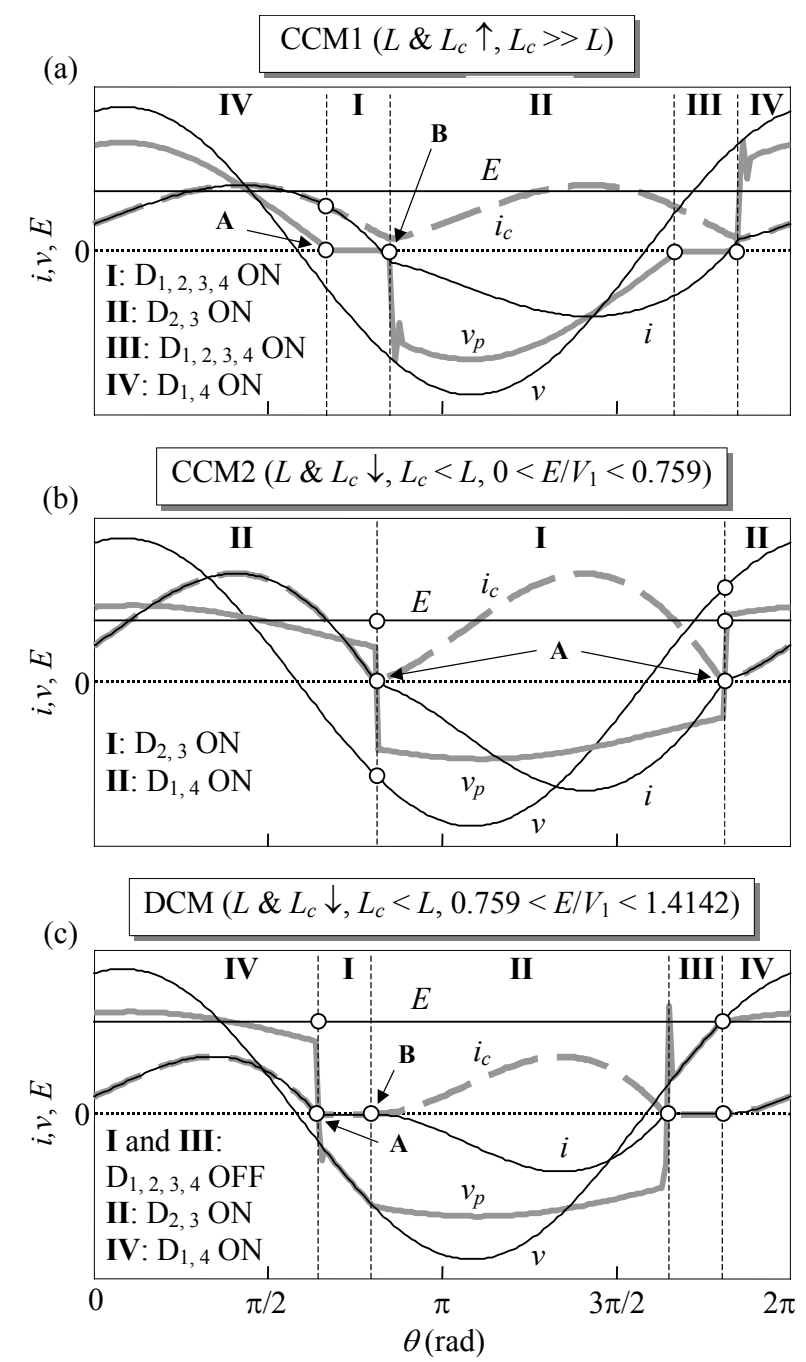

Fig. 2. Conduction modes of EV battery charger equivalent circuit. 


\section{EV Battery Charger Behavior}

Fig. 1 illustrates the typical equivalent circuit of singlephase battery chargers. It consists of a single-phase diode bridge with an AC inductor $L$ (together with its associated resistance $R$ ), the resistance $R_{C}$ and the inductance $L_{C}$ of the charger circuit and the emf $E$ of the battery under charge (together with its internal resistance $R_{E}$ ). According to Fig. 2, this circuit can operate in three different modes:

- Continuous conduction mode 1 (CCM1): For a given ratio $E / V_{1}$, the zero-crossing of the current $i$ occurs after the zero-crossing of the voltage $v_{p}$ [point $\mathrm{A}$ in Fig. 2(a)]. This happens because the circuit inductances, or rather the inductance $L_{C}$, are large enough to hold the current (note that the current $i_{c}$ is held beyond the fall of the current $i$ ). Thus, the open diode pair turns on so that the four diodes are on (segments I and III). The diodes remain on until the zero-crossing of the current $i$ [point B in Fig. 2 (a)]. In this conduction mode, two states can be distinguished: a) The currents $i$ and $i_{c}$ are not the same and flow through the four diodes [segments I and III in Fig. 2 (a)]; b) The currents $i$ and $i_{c}$ are the same and flow through one diode pair, charging the battery [segments II and IV in Fig. 2 (a)].

- Continuous conduction mode 2 (CCM2): For a given ratio $E / V_{1}$, the zero-crossing of the current $i$ [point $\mathrm{A}$ in Fig. 2 (b)] occurs before the natural zero-crossing of the voltage $v_{p}$ because the circuit inductances are smaller than in CCM1 circuit. As the current zero-crossing takes place when the supply voltage is greater than the emf $E$ due to the small ratio $E / V_{1}$, the current continues flowing through the other pair of diodes (i.e., the diodes which are on turn off and the diodes which are off turn on). In this conduction mode, one state can be distinguished: a) The current $i$ flows through the diode pair $\mathrm{D}_{2,3}$ or $\mathrm{D}_{1,4}$ [segment $\mathrm{I}$ or II in Fig. 2 (b), respectively].

- Discontinuous conduction mode (DCM): For a given ratio $E / V_{1}$, the zero-crossing of the current $i$ [point $\mathrm{A}$ in Fig. 2 (c)] occurs before the natural zero-crossing of the voltage $v_{p}$ because the circuit inductances are smaller than in CCM1 circuit. As the current zero-crossing takes place when the supply voltage is smaller than the emf $E$ due to the large ratio $E / V_{1}$, the current does not continue flowing through the diodes (i.e., the diodes which are on turn off). The diodes remain off until the rectified supply voltage is greater than the emf $E$ [point B in Fig. 2 (c)]. In this conduction mode, two states can be distinguished: a) The current $i$ is zero because the diodes are off [segments I and III in Fig. 2 (c)]; b) The current $i$ flows through one diode pair, charging the battery [segments II and IV in Fig. 2 (c)]. CCM2 corresponds to discharge lamp behavior, which has been widely studied and modeled in the literature [13]. CCM1 and DCM are two possible battery charger behaviors, DCM being the most common one. The following section presents the modeling of both conduction modes.

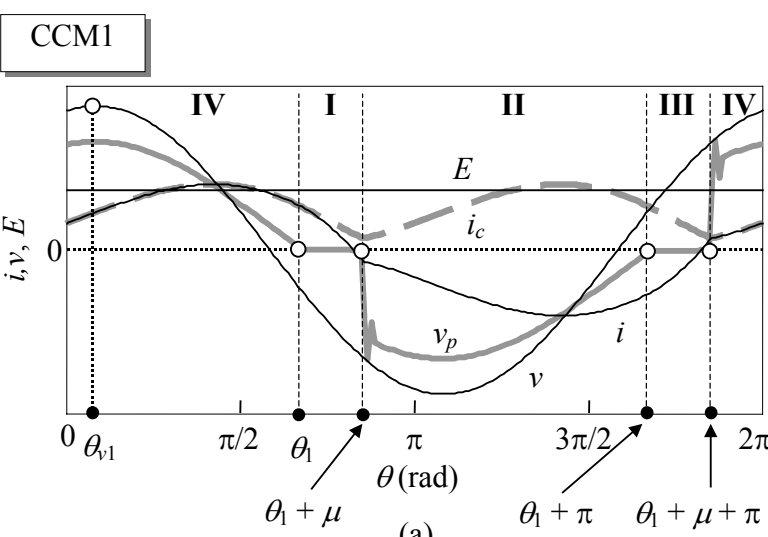

(a)

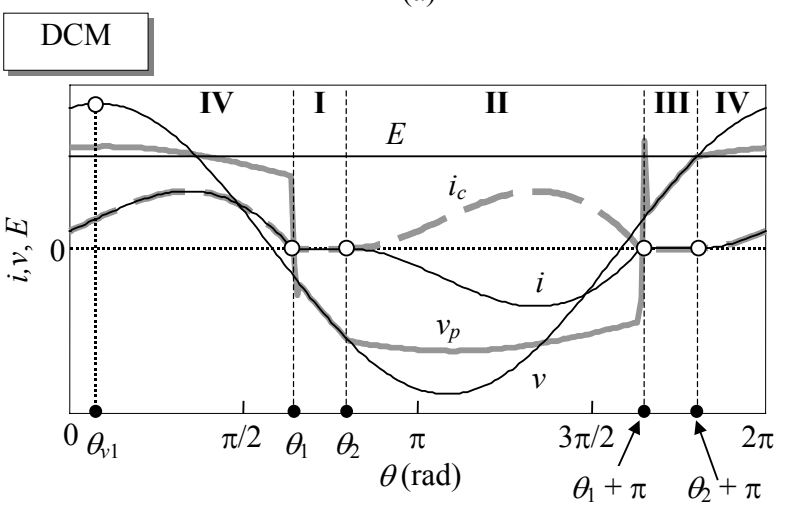

(b)

Fig. 3. EV battery charger modeling.

\section{EV Battery Charger Modeling}

\section{A. CCM1 Modeling}

Fig. 3(a) shows the voltage and current waveforms of the battery charger in CCM1. The commutation angle $\theta_{1}$ and the overlap angle $\mu$, which define charger behavior considering half-wave symmetry conditions, are illustrated in Fig. 3(a). To obtain the instantaneous waveshape of the battery charger currents, such angles must be determined by analyzing the circuit states corresponding to the current segments I and II. Thus, the analysis of the circuit in Fig. 3(a) reveals that the equations characterizing the current waveforms of segment I $\left(\theta_{1}<\theta<\theta_{1}+\mu\right) \quad$ and segment II $\left(\theta_{1}+\mu<\theta<\theta_{1}+\pi\right)$ are

$$
\begin{aligned}
& \text { (I) }:\left\{\begin{array}{l}
R i^{(\mathrm{I})}+X \frac{d i^{(\mathrm{I})}}{d \theta}=v \\
\left(R_{c}+R_{E}\right) i_{c}^{(\mathrm{I})}+X_{c} \frac{d i_{c}^{(\mathrm{I})}}{d \theta}+E=0
\end{array}\right. \\
& \text { (II): }\left\{\begin{array}{l}
R_{t} i^{(\mathrm{II})}+X_{t} \frac{d i^{(\mathrm{II})}}{d \theta}-E=v, \\
i_{c}^{(\mathrm{II})}=-i^{(\mathrm{II})}
\end{array}\right.
\end{aligned}
$$

where $\quad R_{t}=R+R_{c}+R_{E}, \quad X=L \cdot \omega, \quad X_{c}=L_{c} \cdot \omega$, $X_{t}=\left(L+L_{c}\right) \cdot \omega, \quad \omega=2 \pi f$ and $f$ is the fundamental frequency. The expression of these currents is obtained by solving (1) 

(I) $:\left\{\begin{array}{l}i^{(\mathrm{I})}=K^{(\mathrm{I})} e^{-\frac{R}{X} \theta}+g^{(\mathrm{I})}(\theta) \\ i_{c}^{(\mathrm{I})}=K_{c}^{(\mathrm{I})} e^{-\frac{R_{c}+R_{E}}{X_{c}} \theta}-\frac{E}{R_{c}+R_{E}}\end{array}\right.$,
(II) $: i^{(\mathrm{II})}=-i_{c}^{(\mathrm{II})}=K^{(\mathrm{II})} e^{-\frac{R_{t}}{X_{t}} \theta}+g^{(\mathrm{II})}(\theta)+\frac{E}{R_{t}}$,

where

$$
\begin{aligned}
g^{(i)}(\theta) & =\sqrt{2} \sum_{k=1}^{3,5, \ldots} I_{k}^{(i)} \cos \left(k \theta+\beta_{k}^{(i)}\right) \quad(i=\mathrm{I}, \mathrm{II}), \\
I_{k}^{(\mathrm{I})} & =\frac{V_{k}}{\sqrt{R^{2}+(k X)^{2}}}, \quad \beta_{k}^{(\mathrm{I})}=\phi_{v k}-\operatorname{tg}^{-1}\left(\frac{k X}{R}\right), \\
I_{k}^{(\mathrm{II})} & =\frac{V_{k}}{\sqrt{R_{t}^{2}+\left(k X_{t}\right)^{2}}}, \quad \beta_{k}^{(\mathrm{II})}=\phi_{v k}-\operatorname{tg}^{-1}\left(\frac{k X_{t}}{R_{t}}\right) .
\end{aligned}
$$

The expressions of the constants $K^{(\mathrm{I})}, K_{c}^{(\mathrm{I})}$ and $K^{(\mathrm{II})}$ in (2) are obtained from the continuity and periodicity conditions:

$$
\begin{aligned}
& i^{(\mathrm{II})}\left(\theta_{1}+\mu\right)=i^{(\mathrm{I})}\left(\theta_{1}+\mu\right), \\
& i^{(\mathrm{I})}\left(\theta_{1}\right)=-i^{(\mathrm{II})}\left(\theta_{1}+\pi\right), \\
& i_{c}^{(\mathrm{I})}\left(\theta_{1}\right)=i_{c}^{(\mathrm{II})}\left(\theta_{1}+\pi\right)=-i^{(\mathrm{II})}\left(\theta_{1}+\pi\right) \\
& \Rightarrow\left[\begin{array}{ccc}
e^{-\frac{R}{X}\left(\theta_{1}+\mu\right)} & -e^{-\frac{R_{t}}{X_{t}}\left(\theta_{1}+\mu\right)} & 0 \\
e^{-\frac{R}{X} \theta_{1}} & e^{-\frac{R_{t}}{X_{t}}\left(\theta_{1}+\pi\right)} & 0 \\
0 & e^{-\frac{R_{t}}{X_{t}}\left(\theta_{1}+\pi\right)} & e^{-\frac{R_{c}+R_{E}}{X_{c}} \theta_{1}}
\end{array}\right]\left[\begin{array}{c}
K^{(\mathrm{I})} \\
K^{(\mathrm{II})} \\
K_{c}^{(\mathrm{I})}
\end{array}\right]= \\
& =\left[\begin{array}{c}
-g^{(\mathrm{I})}\left(\theta_{1}+\mu\right)+g^{(\mathrm{II})}\left(\theta_{1}+\mu\right)+\frac{E}{R_{t}} \\
-g^{(\mathrm{I})}\left(\theta_{1}\right)+g^{(\mathrm{II})}\left(\theta_{1}\right)-\frac{E}{R_{t}} \\
\frac{E}{R_{c}+R_{E}}+g^{(\mathrm{II})}\left(\theta_{1}\right)-\frac{E}{R_{t}}
\end{array}\right] .
\end{aligned}
$$

The commutation and overlap angles are obtained by solving the error function non-linear system of the form $\mathbf{F}(\mathbf{x})=0$ derived from the symmetry and segment change conditions:

$$
\begin{aligned}
& f_{1}(\mathbf{x})=v_{p}^{(\mathrm{II})}\left(\theta_{1}+\pi\right)=0 \\
& f_{2}(\mathbf{x})=i^{(\mathrm{I})}\left(\theta_{1}+\mu\right)+i_{c}^{(\mathrm{I})}\left(\theta_{1}+\mu\right)=0,
\end{aligned}
$$

where $\mathbf{F}=\left(f_{1}, f_{2}\right), \mathbf{x}=\left(\theta_{1}, \mu\right)$ and

$$
v_{p}^{(\mathrm{II})}=v-R i^{(\mathrm{II})}-X \frac{d i^{(\mathrm{II})}}{d \theta} .
$$

\section{B. DCM Modeling}

Fig. 3(b) shows the voltage and current waveforms of the battery charger in DCM. The commutation angles $\theta_{1}$ and $\theta_{2}$, which define charger behavior considering half-wave symmetry conditions, are illustrated in Fig. 3(b). To obtain the instantaneous waveshape of the battery charger currents, these angles must be determined by analyzing the circuit states corresponding to the current segments I and II. Thus, the analysis of Fig. 3(b) reveals that the equations characterizing the current waveforms of segment I $\left(\theta_{1}<\theta<\theta_{2}\right)$ and segment II $\left(\theta_{2}<\theta<\theta_{1}+\pi\right)$ are

$$
\begin{aligned}
& (\mathrm{I}): i^{(\mathrm{I})}=0, \quad i_{c}^{(\mathrm{I})}=0, \\
& (\mathrm{II}): R_{t} i^{(\mathrm{II})}+X_{t} \frac{d i^{(\mathrm{II})}}{d \theta}-E=v, \quad i_{c}^{(\mathrm{II})}=-i^{(\mathrm{II})},
\end{aligned}
$$

where $R_{t}=R+R_{c}+R_{E}, X_{t}=\left(L+L_{c}\right) \cdot \omega$. The expression of these currents is obtained by solving (7):

$$
\begin{aligned}
& (\mathrm{I}): i^{(\mathrm{I})}=i_{c}^{(\mathrm{I})}=0, \\
& (\mathrm{II}): i^{(\mathrm{II})}=-i_{c}^{(\mathrm{II})}=K^{(\mathrm{II})} e^{-\frac{R_{t}}{X_{t}} \theta}+g^{(\mathrm{II})}(\theta)+\frac{E}{R_{t}},
\end{aligned}
$$

where

$$
\begin{aligned}
& g^{(\mathrm{II})}(\theta)=\sqrt{2} \sum_{k=1}^{3,5, \ldots} I_{k}^{(\mathrm{II})} \cos \left(k \theta+\beta_{k}^{(\mathrm{II})}\right), \\
& I_{k}^{(\mathrm{II})}=\frac{V_{k}}{\sqrt{R_{t}^{2}+\left(k X_{t}\right)^{2}}}, \quad \beta_{k}^{(\mathrm{II})}=\phi_{v k}-\operatorname{tg}^{-1}\left(\frac{k X_{t}}{R_{t}}\right) .
\end{aligned}
$$

The expression of the constant $K^{(\mathrm{II})}$ in (8) is obtained from the continuity condition:

$$
\begin{aligned}
& i^{(\mathrm{II})}\left(\theta_{2}\right)=i^{(\mathrm{I})}\left(\theta_{2}\right)=0 \\
& \Rightarrow \quad K^{(\mathrm{II})}=-e^{\frac{R_{t}}{X_{t}}\left(\theta_{2}\right)}\left(\frac{E}{R_{t}}+g^{(\mathrm{II})}\left(\theta_{2}\right)\right) .
\end{aligned}
$$

The commutation angles are obtained by solving the error function non-linear system of the form $\mathbf{F}(\mathbf{x})=0$ derived from the symmetry and segment change conditions

$$
\begin{aligned}
& f_{1}(\mathbf{x})=v\left(\theta_{2}\right)+E=0 \\
& f_{2}(\mathbf{x})=i^{(\mathrm{II})}\left(\theta_{1}+\pi\right)=0,
\end{aligned}
$$




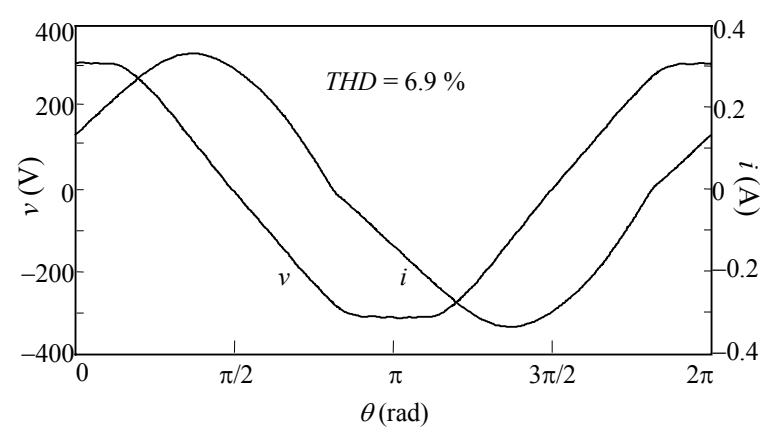

Fig. 4. Measured current waveforms of CCM2.

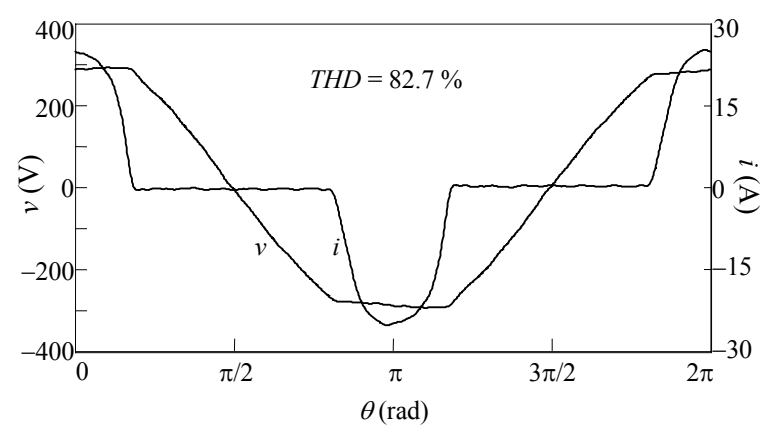

Fig. 5. Measured current waveforms of DCM.

where $\mathbf{F}=\left(f_{1}, f_{2}\right)$ and $\mathbf{x}=\left(\theta_{1}, \theta_{2}\right)$.

\section{EV Battery Charger Model Validation}

\section{A. Experimental measurements}

Three examples of current waveforms are shown to report the current conduction modes in Fig. 2:

- Figure 3 in [7] illustrates the CCM1 current waveforms. They correspond to measurements of a $480 \mathrm{~V}$ battery charger connected in a $240 \mathrm{~V}$ supply installation.

- Fig. 4 illustrates the CCM2 current waveform. It corresponds to measurements of a SYLVANIA $26 \mathrm{~W}$ compact fluorescent lamp (Lynx D CF-D 26 W/840 model) installed in a NEXIA $230 \mathrm{~V} / 50 \mathrm{~Hz} 2 \times 26 \mathrm{~W}$ Serie 01 downlight (G24d-3 model) in a household installation.

- Fig. 5 illustrates the DCM current waveform. It corresponds to measurements of a Piaggio Porter EV $2.2 \mathrm{~kW}$ battery charger in a garage installation.

The total harmonic distortion of the currents

$$
T H D(\%)=100 \frac{\sqrt{\sum_{k=2}^{\infty} I_{k}^{2}}}{I_{1}}
$$

is also provided in the previous figures. The measurements of the Fig. 4 and Fig. 5 were made with the supply network analyzer AR5-L of CIRCUTOR.
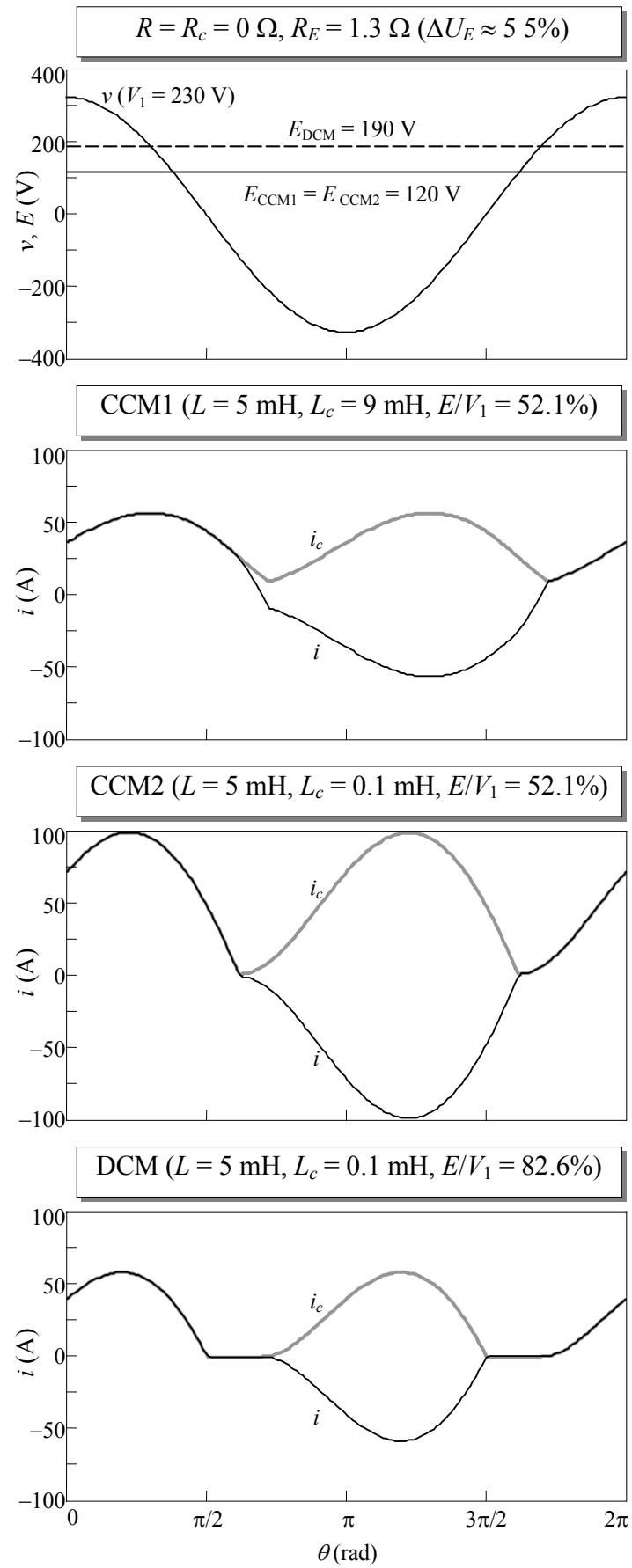

Fig. 6. Simulated current waveforms of the conduction modes of the battery charger equivalent circuit.

\section{B. Numerical simulations}

Numerical simulations were performed with PSpice software to study the current conduction modes in Fig. 2. The obtained waveforms are illustrated in Fig. 6. The parameters of the equivalent circuits (Fig. 1) corresponding to the different simulations are also shown in Fig. 6. The conduction modes analysis in Section II can be contrasted with the results in Fig. 6. The experimental measurements in Section 4.A could be reproduced with the studied models. To this end, first the parameter models must be estimated from measurements. This must be studied in detail in further investigations. 


\section{Conclusion}

The paper presents the equivalent circuit of EV battery chargers and analyzes its time behavior, which has three different current conduction modes (CCM1, CCM2 and DCM). CCM2 corresponds to discharge lamp behavior and CCM1 and DCM correspond to battery charger behavior. The analytical model of CCM1 and DCM is studied in detail and the equations characterizing both conduction modes are provided. The three current conduction modes of the battery charger equivalent circuit are illustrated with experimental measurements and PSpice simulations.

\section{Acknowledgement}

The authors would like to thank the PFC Division Technical Department of CIRCUTOR S.A. for its contribution to this paper with the battery charger measurements.

This research is carried out with the financial support of grant DPI2010-15448, which the authors gratefully acknowledge.

\section{References}

[1] A. Perujo and B. Ciuffo, "Potential impact of electric vehicles on the electric supply system: A case study for the province of Milan, Italy", Joint Research Center, Institute for Environment and Sustainability, European Commission, 2009.

[2] E. Valsera-Naranjo, A. Sumper, P. Lloret-Gallego, R. Villafáfila-Robles and A. Sudria-Andreu, "Electrical vehicles: State of art and issues for their connection to the network", in Proc. of the $10^{\text {th }}$ International Conference on Electrical Power Quality and Utilisation (EPQU 2009) 2009, pp. 60-62.

[3] G. A. Putrus, P. Suwanapingkarl, D. Johnston, E. C. Bentley and M. Narayana, "Impact of electric vehicles on power distribution networks", in Proc. of the Vehicle Power and Propulsion Conference (VPPC 2009) 2009, pp. 827-831.

[4] J. A. Orr, A. E. Emanuel and K. W. Oberg, "Current harmonics generated by a cluster of electric vehicle battery chargers", IEEE Trans. on Power Apparatus and Systems, Vol. PAS-101, No. 3, March 1982, pp. 691-700.

[5] J. A. Orr, A. E. Emanuel and D. J. Pileggi, "Current harmonics, voltage distortion, and powers associated with battery chargers. Part I: Comparisons among different types of chargers", IEEE Trans. on Power Apparatus and Systems, Vol. PAS-101, No. 8, August 1982, pp. 27032710.

[6] J. A. Orr, A. E. Emanuel and D. J. Pileggi, "Current harmonics, voltage distortion and powers associated with electric vehicle battery chargers distributed on the residential power system", IEEE Trans. on Industry Applications, Vol. IA-20, No. 4, July/August 1984, pp. 727-734.

[7] P. T. Staats, W. M. Grady, A. Arapostathis and R. S. Thallam, "A statistical method for predicting the net harmonic currents generated by a concentration of electric vehicle battery chargers", IEEE Trans. on Power Delivery, Vol. 12, No. 3, July 1997, pp. 1258-1266.

[8] P. T. Staats, W. M. Grady, A. Arapostathis and R. S. Thallam, "A statistical analysis of the effect of electric vehicle battery charging on distribution system harmonic voltages", IEEE Trans. on Power Delivery, Vol. 13, No. 2, April 1998, pp. 640-646.

[9] P. T. Staats, W. M. Grady, A. Arapostathis and R. S. Thallam, "A procedure for derating a substation transformer in the presence of widespread electric vehicle battery charging", IEEE Trans. on Power Delivery, Vol. 12, No. 4, October 1997, pp. 1562-1568.

[10] Y. J. Wang, R. M. O'Connell and G. Brownfield, "Modeling and prediction of distribution system voltage distortion caused by nonlinear residential loads", IEEE Trans. on Power Delivery, Vol. 16, No. 4, October 2001, pp. 744-751.

[11] T. A. Buchh and A. Domijan Jr., "Harmonic effect of electric vehicle loads", Int. Journal of Power and Energy Systems, Vol. 21, No. 2, 2001, pp. 62-66.

[12] J. C. Gómez and M. M. Morcos, "Impact of EV battery chargers on the power quality of distribution systems", IEEE Trans. on Power Delivery, Vol. 18, No. 3, July 2003, pp. 975-981.

[13] J. J. Mesas, L. Sainz and A. Ferrer, "Deterministic and stochastic assessment of the harmonic currents consumed by discharge lamps", Electric Power Systems Research, Vol. 81, No. 1, January 2011, pp. 10-18. 